\title{
MODELLING PN FORMATION FROM HYDRODYNAMICS AND RADIATION
}

\author{
G. MELLEMA
}

Sterrewacht Leiden, P.O. Box 9513, 2300 RA Leiden, The Netherlands

The formation of planetary nebulae from the mass loss remnants of previous phases forms an interesting problem in both hydrodynamics and astrophysics. Not in the least because of the asphericity that most PNe show. The origin of this asphericity is still largely a mystery. Binarity is the most favoured explanation at the moment (Morris 1987)

By assuming that the asphericity is introduced by some cause in the AGB phase, the work of Soker \& Livio 1989, Mellema et al. 1991, and Icke et al. 1992 has shown that even if the effects of radiation from the central star and the gas are neglected (adiabatic case), many interesting features resembling observed aspherical PNe are found. See the paper of Icke at this conference for an overview of this. This paper reports on the work of including radiation effects in these models.

The essence of the problem of PN formation is that both hydrodynamics and radiation play an important role. Furthermore, the radiation is what we observe. That is why we have modified our $2 \mathrm{D}$ hydrocode such that the hydrodynamics and the radiation and ionization state are solved at the same time. It is important to note that we treat the problem consistently this way. That is, the program calculates the radiation losses, which influence the flow, and which are also direct observables. This allows us to produce images of our models in the well-known emission lines of $\mathrm{H} \alpha,[\mathrm{OIII}]$, and [NII]. Combined with the velocity information from the hydrodynamics, kinematic data can also be constructed.

This combined radiation and hydrodynamics code should be able to answer some of the questions regarding PNe formation. We plan to use it to simulate the emergence of $\mathrm{PNe}$, especially concentrating on the role of the different shaping components. These are the (slow) mass loss on the AGB, and the (fast) one after that. It should be possible to place limits on the behaviour of the various winds, and separate influence of the wind interaction from the effects of ionization fronts. The possibility to construct both synthetic images and kinematic data should be a great help in comparing to the observational data.

All this is work done together with Adam Frank, Bruce Balick, and Vincent Icke whose contributions at these proceedings touch upon some other aspects of this project.

\section{References}

Icke, V., Frank, A., Balick B. (1992) A\&A 253, 244.

Mellema, G., Eulderink, F., Icke V. (1991) A\&A 252, 719.

Morris, M. (1987) Publ. Astr. Soc Pac. 99, 1115.

Soker, N. and Livio, M. (1989) ApJ 339, 268. 